Aus dem Laboratorium der Hydrotherapeutischen Anstalt der Universität in Berlin (Leiter: Geh. Med.-Rat Prof. Dr. L. Brieger).

\title{
Ueber das Wesen, die Wirksamkeit und Haltbarkeit des nach der Briegerschen Schüttelmethode hergestellten Typhus- schutzstoffes.
}

Von Oberstabsarzt z. D. Dr. R. Bassenge.

Vor zwei Jahren wurde vom Verfasser dieser Arbeit und M. Mayer eine Methode der Typhusschutzimpfung bekannt gegeben, welche es ermöglicht, durch eine einmalige Finspritzung von $2 \mathrm{ccm}$ eines durchaus klaren, niederschlagsfreien Toxins einen länger dauernden Schutz gegen Typhusinfektion auszuüben. Das Wesen dieses nach der Briegerschen Methode hergestellten Toxins besteht darin, dab es durch 24 stündiges Schütteln der lebenden Typhusbazillen in destilliertem Wasser bei Zimmertemperatur gewonnen wird. Jeder dieser Punkte ist von Bedeutung; auch ist es unerläßlich, zur Bereitung des Toxins Typhusstämme von hoher und konstanter Virulenz zu verwenden, um ein Präparat von gleicher Wirksamkeit zu erhalten.

Die Herstellung des Schutzstoffes gestaltete sich folgendermaßen:

Ein auf seine Virulenz geprüfter Typhusstamm wurde auf $2 \%$ \% Röhrchen, mit gleichen Nährbodenmengen in breiter Fläche 24 Stunden im Brutschrank von $37^{\circ} \mathrm{C}$ Temperatur so gezüchtet, daß die ganze Agarfläche von einer gleichmäßigen Schicht Typhuskultur bedeckt war. Je zehn solcher Typhuskulturen wurden mit $50 \mathrm{ccm}$ sterilen destillierten Wassers abgeschwemmt und noch weiterhin mit $5 \mathrm{ccm}$ sterilen destillierten Wassers nachgespült, sodaß die Kulturmasse vollkommen aufgenommen wurde. Die auf diese Weise erhaltenen etwa $56-57 \mathrm{ccm}$ Kulturaufschwemmung wurden in braunen Erlenmeyerschen Kölbchen 24 Stunden bei Zimmertemperatur dem Schüttelapparat übergeben und danach im Pukallfilter bakterienfrei filtriert. Das Filtrat wurde im Vakuum auf $20 \mathrm{ccm}$ eingeengt, auf Bakterienfreiheit geprüft und in Glasröhrchen eingeschmolzen. Es entsprachen dann $2 \mathrm{ccm}$ des Filtrats genau dem Extrakt einer Kultur. Man läßt zweckmäßig so viel Wasser vom Vakuum verdampfen, daß etwas weniger als $20 \mathrm{ccm}$ Filtrat in der graduierten Schale zurückbleiben; man füllt dann mit sterilem destilliertem Wasser bis auf $20 \mathrm{ccm}$ auf und ermöglicht so eine ganz genaue Dosierung. Man kann auch zur Wiederherstellung der Menge von $20 \mathrm{ccm}$ des eingedickten Toxins eine Phenollösung in einer Stärke verwenden, sodaß das Toxin etwa $0,2-0,3 \%$ Phenol enthält. Seine Wirksamkeit wird dadurch nicht beeinträchtigt. Das Schüitteln der Bakterien erfolgt in Kolben von gefärbtem Glase; auch die Glocke des Vakuums besteht aus gefärbtem Glase. Auf diese Weise erhält man ein Präparat von einer konstanten Wirksamkeit und einer gleichen Konzentration, wie zahlreiche Refraktometerbestimmungen des Toxins erwiesen haben. Die Gleichmäßigkeit des Präparates ließ sich direkt durch die Bestimmung der Brechungsexponenten mit dem Refraktometer nachweisen.')

Da durch das Pukallfilter in den Filterporen wirksame Stoffe zurückgehalten werden können, empfiehlt es sich, zur Filtration nur frisch im Dampftopf sterilisierte Filter zu verwenden und unmittelbar vor und nach dem Filtrieren eine kleine Menge sterilen destillierten Wassers durch das Filter zu saugen, sodaß das Filter frei von Toxin wird.

Durch die geschilderte Herstellungsweise ist die Brieger sche Forderung der möglichst schonenden Extraktion des Toxins

1) Genauere Mitteilungen über diese Bestimmungen sowie über solche von Seris und Schutzstoffen auf physikalisch-chemischem Wege werden in der nächsten Zeit von R. Bassenge und M. Krause veröffentlicht werden. 
aus den lebenden Bakterien erfüllt. Den Filterrückstand bilden ungeheure Mengen außerordentlich lebhaft beweglicher Typhusbazillen, welche im hängenden Tropfen unter dem Mikroskop das Bild eines Mückenschwarms darbieten, ähnlich wie es sich auch beim Betrachten einer Cholerabazillenaufschwemmung im hängenden Tropfen ergibt. Es scheint, als ob durch diese Behandlungsmethode, sei es nun die 24 stündige Ausschüttelung, sei es der Aufenthalt der Bakterien in der anisotonen Flüssigkeit, oder sei es schlieblich die Saugwirkung der an den Pukall angeschlossenen Wasserstrahlpumpe, die Bakterien zu ganz besonders starker Beweglichkeit angeregt werden. Niemals erhält man in einem hängenden Tropfen Typhuskultur, $a b$ dieser nun aus Bouillon besteht, oder ob man Agarkultur in physiologischer Kochsalzlösung aufschwemmt, selbst. wenn man sehr große Mengen Typhusbazillen für die Aufschwemmung verwendet, ein solches Bild herumschwirrender Bazillen.

Das Toxin enthält noch genügende Mengen Eiweißstoffe und Salze für ein weiteres Wachstum der Typhusbazillen, trotzdem das Toxin aus einer Aufschwemmung der Bazillen in destilliertem Wasser gewonnen wird. Wenn man solches bakterienfreies, klares Toxin aufs neue mit Typhusbakterien impft und dasselbe für drei Tage dem Brutschrank von $37^{\circ} \mathrm{C}$ übergibt, so vermehren sich die Typhusbakterien so reichlich, daß man von neuem eine Ausschüttelung der Bazillen zur Gewinnung eines stärkeren, konzentrierteren Giftstoffes gewinnen kann. In den ersten 24 Stunden ist das Wachstum nicht besonders intensiv, ja es findet sogar ein Absterben einer Anzahl von Typhusbazillen statt. Nach 2-3 Tagen scheint aber eine Gewöhnung an den anisotonen Nährboden einzutreten, wodurch dann das weitere Wachstum bedingt wird.

Das so gewonnene Toxin kann man wiederum mit Typhusbazillen beschicken, drei Tage als Nährboden verwenden, die Bakterien ausschütteln und daraus durch $\mathrm{Pukallfiltration}$ sich neues Toxin herstellen. Selbst wenn man dieses Verfahren monatelang $15-20$ mal wiederholt, beobachtet man immer noch ein allerdings schwächer werdendes Wachstum der Typhusbazillen.

In gleicher Weise wurde gewöhnliche Rinderbouillon als Nährboden verwendet, in dieser die Typhusbazillen ausgeschüttelt, dann durch Filtrieren von den Bazillen befreit, und das bakterienfreie. Filtrat aufs neue mit Typhusbazillen beschickt. Zum Vergleich wurde auch dieses Verfahren Monate hindurch fortgesetzt, ohne dal die Nährbouillon erheblich Einbuße erlitt an ihrer Fähigkeit, die Typhusbazillen weiter zu kultivieren. Allerdings wuchsen diese in dem Parallelversuch, in dem Bouillontoxin stets reichlicher als in dem Wassertoxin. Das konnte sowohl durch die makroskopische Beobachtung der Trübung des Nährbodens als auch durch die mikroskopische' Schätzung der Beobachtung im hängenden Tropfen nachgewiesen werden; auch wurde diese eigentlich selbstverständliche Tatsache gelegentlich noch festgestellt durch Aussaat abgemessener Mengen der gezüchteten Kulturen und direkte Zählung der entwickelten Keime.

Von maßgeblicher Seite ist die Vermutung ausgesprochen worden, daß die Wirksamkeit des durch die Briegersche Schüttelmethode gewonnenen Typhustoxins zuweilen dem Umstande zuzuschreiben sei, daß vereinzelte lebende Typhusbazillen die Filterporen des $P$ ukallfilters oder Sprünge durchdrängen und so eine aktive Immunisierung der damit behandelten Personen mit vereinzelten lebenden Typhusbakterien zustande komme. Diese Vermutung wird durch den vorher beschriebenen Versuch vollkommen hinfällig, da durch denselben nachgewiesen ist, daß in dem Filtrat die Typhusbakterien gute Existenzbedingungeu für ihre Weiterentwicklung haben. Diese Weiterentwicklung der Typhusbakterien würde sich sofort in dem Typhustoxin makroskopisch durch eine deutliche Trübung des Toxins kennzeichnen.

Die Hoffnung, ein erheblich wirksameres, konzentrierteres Toxin durch immer wieder neue Beschickung des Filtrats mit Typhusbazillen, Bebrütung und Ausschüttelung zu gewinnen, hat sich nicht verwirklicht. Die Ergebnisse dieser Methode waren schwankende; auch ist die Herstellungsweise zu umständlich, um zurzeit eine Bedeutung für die praktische Ausnützung $\mathrm{zu}$ gewinnen.

Die Anregung, auf diese Weise ein stärkeres Toxin zu gewinnen, indem die Typhusbazillen immer von neuem Gift an den bereits stark gifthaltigen Nährboden abgeben, stammt von Brieger, wie überhaupt die praktische Verwirklichung der Gewinnung eines Typhustoxins den Arbeiten dieses Forschers zu danken ist.

Brieger verwendete zuerst im Jahre 1892 mit Kitasato und Wassermann Typhusbazillen, die auf Thymusbouillon gezïchtet und bei $60^{\circ} \mathrm{C}$ abgetötet waren, zur Immunisierung von Versuchstieren. Auch war Brieger der erste, welcher im Verein mit Karl Fraenkel im Jahre 1890 schon Toxine aus Typhusbazillenkulturen herstellte, die durch Chamberland-Tonfilter bakterienfrei gemacht worden waren. Versuche am Menschen mit abgetöteten Typhusbakterien-Aufschwemmungen wurden in Deutschland zuerst von Eugen Fraenkel 1893 und in England von Wright zuerst im Juli und August 1896 gemacht. Während in den neueren Arbeiten über Schutzimpfung gegen Typhus diese Priorität nicht vollguiltig anerkannt wird, äußert sich Wright selbst über seine Prioritätsansprüche betreffend die Verwendung des Typhusschutzstoffes wörtlich folgendermaßen ${ }^{1}$ ): "Our first vaccinations against typhoid were undertaken in the months of July and August of last year. These vaccinations were put on record by one of us in the Lancet on Sept. 19th 1896 in a paper which dealt primarly with the question of serous haemorrhage. A reprint of this paper was sent among others to Professor Pfeiffer. Nearly two months after the date of this paper Prof. Pfeiffer published in conjunction with Dr. Kolle a paper on two cases of typhoid vaccination. The method of vaccinations which these authors have adopted, is exactly similar to the one that we had previously adopted. Like our own method, it was based upon the methods which have been so succesfully employed by Mr. Haffkin in his anticholera inoculations."

$\mathrm{Zu}$ dieser kurzen historischen Abschweifung sei noch bemerkt, daß Wright zu seinen ersten Impfungen Typhusagarkulturen benutzte, welche mit abgemessenen Mengen Bouillon abgeschwemmt und in kalibrierten Pipetten (tubes) bei $60^{\circ}$ abgetötet wurden. Die Dosierung wurde nach der Bazillenzahl und dem Grade der Virulenz der benutzten Typhuskulturen bestimmt. Erst später zur Herstellung des Impfstoffes für Massenimpfungen benutzte Wright Kulturen aus Bouillonkolben, die ebenfalls bei $60^{\circ} \mathrm{C}$ abgetötet wurden. Daß diese Temperatur erreicht war, wurde auf sinnreiche Weise durch einen mit Paraffin verschlossenen GJasschwimmer festgestellt. Das dazu verwendete Paraffin hatte einen Schmelzpunkt von $60^{\circ}$. Daß dann Wright schon vom Jahre 1897 an mit Massenimpfungen bei dem Pflegerpersonal des Barming Asylum in Maidstone begonnen und dieselben in den darauf folgenden Jaliren bei vielen Tausenden Soldaten der indischen, ägyptischen und südafrikanischen Armee fortgesetzt hat, dürfte auch in Deutschland bekannt sein.

Das Briegersche „Schütteltoxin“, wie wir es kurz bezeichnen wollen, im Gegensatz zum alten Briegerschen Toxalbumin, das aus abgetöteten, filtrierten Typhusbouillonkulturen hergestellt wurde und im Gegensatz zu den aus abgetöteten Bouillonkulturen oder in Kochsalzlösung aufgeschwemmten Agarkulturen - ist ein Toxin, welches durch die schonendste Extraktion aus lebenden Typhusbakterien gewonnen wird. Es enthält derartige Mengen wirksame Antikörper bildende Substanzen, daß die einmalige Injektion des aus einer Typhusagarkultur gewonnenen Impfstoffes genügt, um für längere Zeit einen Schutz gegen Typhusinfektion auszuüben. Der mit der einmaligen Injektion erreichte Wert der Schutzstoffe des Blutserums der mit Toxin behandelten Personen ist für gewöhnlich so groß, dab $0,01 \mathrm{ccm}$ des Serums Meerschweinchen von etwa $200 \mathrm{~g}$ Gewicht auch bei Injektion einer sechs- bis zehnmal tödlichen Dosis virulenter Typhusbazillen vor dem sicheren Tode erretten, so grob, dab bei solchen Tieren für gewöhnlich gar keine Krankheitserscheinungen auftreten, während Kontrolltiere meist innerhalb 8-12 Stunden nach der Injektion zugrunde gehen. Da hierbei sehr grobe Mengen Typhusbakterien den Tieren eingespritzt werden, ist man zu dem Schlub berechtigt, daß in dem Serum der mit dem Schütteltoxin behandelten Personen nicht nur bakteriolytische, sondern auch antitoxische Stoffe in grober Menge vorhanden sind.

Immerhin läßt sich noch nicht mit Sicherheit behaupten, daf das Schütteltoxin das reine Typhusgift ist und ob nicht in das Filtrat auch noch Stoffe übergehen, die mit der Immunisierung vielleicht nichts zu tun haben, sie möglicherweise so-

1) Vgl. A. E. Wright und D. Semple, Remarks on vaccination against typhoid fever. Brit. medical Journal 1897, L. 256 . 
gar beeinträchtigen können. Auch die vollkommene Eintrocknung des Toxins bis zur Pulverform und Wiederauflösung in geringen Mengen Wassers hat darüber einen sicheren Aufschluß nicht $\mathrm{zu}$ geben vermocht.

Das Bestreben, die Schutzimpfung so zu gestalten, daß die geimpften Personen möglichst wenig Beschwerden von derselben haben, möglichst geringe allgemeine und lokale Reaktionen zeigen, hat schon früher veranlaßt, das anisotone Toxin durch entsprechenden Kochsalzzusatz für das menschliche Gewebe isoton zu machen. Es ergab sich aber hierbei kein in die Augen fallender Unterschied. Alle Erfahrungen der Immunisierung gegen Infektionskrankheiten sprechen dafür, daß eine Immunisierung nur durch die danach eintretenden Reaktionen zustande kommt. Das vollständige Ausbleiben einer Reaktion nach der Typhustoxininjektion wäre demnach nicht einmal wünschenswert. Nur muß man unterscheiden zwischen der Reaktion, die das Typhusgift selbst hervorruft, und derjenigen Reaktion, welche durch Einspritzung von Stoffen entsteht, welche dem Typhusgift noch anhaften und mit der Immunisierung gegen Typhus nichts zu tun haben.

Da man aus den Typhusbakterien ohne Schädigung ihrer Lebenseigenschaften und ohne Schädigung ihrer Zellmembranen ein wirksames Typhusgift erhält, so ist der Schluß berechtigt, daß die abgetöteten Typhusbakterien, ihre Zellmembranen und alle diejenigen autolytischen Produkte, welche durch längeren Aufenthalt der abgetöteten Typhusbazillen in der Kochsalzaufschwemmung entstehen, nur einen geringen oder gar keinen Anteil an der Typhusimmunisierung haben, daß sie im Gegenteil diejenigen Stoffe sind, die nutzlos schwere Allgemein- und Lokalreaktionen nach der Injektion auslösen.

Die Herstellung des Typhusschutzstoffes aus abgetöteten Typhusbazillen wird so vorgenommen, daB die auf Agar gezüchteten und in Kochsalzlösung abgeschwemmten Typhusbazillen während einer bis zwei Stunden im Schüttelapparat bei $60^{\circ} \mathrm{C}$ abgetötet werden. Hierbei müssen die Typhusbakterien, solange sie noch am Leben sind, an die Aufschwemmung Stoffe abgeben, die auch im Schütteltoxin enthalten sind. Der fertiggestellte Typhusschutzstoff enthält aber außerdem noch die Bazillenleiber, und dazu treten ferner noch bei längerer Aufbewahrung autolytische Produkte dieser Bazillenleiber. $\mathrm{Er}$ ist einer dauernden Veränderung unterworfen. Der Zusatz von Phenol garantiert seine Haltbarkeit nur bezüglich der Sterilität, nicht bezüglich seiner biochemischen Zusammensetzung. Längere Zeit aufbewahrte Gläschen mit Typhusschutzstoff aus abgetöteten Bazillenaufschwemmungen zeigen am Boden eine weißliche Masse ausgefällter Bazillenleiber, die sich durch Schütteln nicht völlig entfernen und nur durch Abkratzen mit einem Instrument sich wieder in die Flüssigkeit zurückbringen lassen. Aus diesen Gründen ist für die Immunisierung nur frisch bereiteter Impfstoff brauchbar, da älterer längere Zeit aufbewahrter, ganz unkontrollierbare Zerfallsprodukte enthalten muß.

Der Gehalt des Typhusimpfstoffes an abgetöteten Bazillen und Zellmembranen macht es auch erklärlich, daß zunächst recht heftige lokale Reaktionen nach den Injektionen auftreten müssen und, daß ein von diesen reizenden Bestandteilen befreiter Impfstoff viel geringere Reaktionserscheinungen macht und daher viel schneller vom Körper resorbiert wird.

Eignet sich der aus abgetöteten Typhusbazillen bestehende Impfstoff schon aus den oben mitgeteilten Gründen nicht für längere Aufbewahrung, so macht ihn auch die Unmöglichkeit, sich durch makroskopische Prüfung von der Sterilität zu überzeugen, hierfür ungeeignet. Die trübe, milchige Flüssigkeit kann auch bei sorgfältigster Zubereitung infolge Beschädigung des Verschlusses beim Transport durch Zutritt von Bakterien infiltriert werden, ohne daß die Möglichkeit besteht, eine solche Verunreinigung zu erkennen.

Das ist beim Briegerschen Schütteltoxin gänzlich ausgeschlossen. Dasselbe bleibt klar, auch bei mehr als zweijähriger Aufbewahrung, verändert sich nicht im geringsten und zeigt dieselbe Wirksamkeit wie unmittelbar nach der Herstellung. Im Jahre 1905 wurden einige Röhrchen des Schütteltoxins an die Kaiserliche Marine zu Versuchszwecken abgegeben. Seitens des Herrn Generalstabsarztes der Marine Dr. Schmidt wurden zunächst Versuche über die Haltbarkeit des Schutzstoffes angeordnet. Von den Röhrchen, welche diesem Versuche ausgesetzt wurden, wurde eines an Bord S. M. S. Schulschiff "Stosch“, ein anderes an Bord S. M. S. Kreuzer "Sperber“ ein halbes und ein und einhalbes Jahr in subtropischen und tropischen Gegenden Ostasiens, Ost- und Westafrikas und des Atlantischen Ozeans ohne besondere Vorsichtsmabregeln in der Schiffsapotheke aufbewahrt. Der Inhalt beider Röhrchen erschien makroskopisch ebenso unverändert wie das seit derselben Zeit im Eisschrank des Laboratoriums aufbewahrte Schütteltoxin, klar, hellgelb, ohne Trübung und Niederschlag. Es konnte unbedenklich zur Prüfung seiner Wirksamkeit zur Immunisierung verwendet werden. Das in den zugeschmolzenen Röhrchen enthaltene Toxin war bei der im Dezember v. J. und Februar d.J. vorgenommenen Prüfung etwa ein_Jahr acht Monate alt.

Von jedem der beiden Röhrchen wurden Proben von zwei ccm entsprechend dem aus einer Typhusagarkultur gewonnenen Gift der Wärterin W. und dem Wärter L. subkutan injiziert. Die nach der Injektion eintretenden Lokalrelaktionen waren außerordentlich gering. Bei der Wärterin trat eine Allgemeinreaktion überhaupt nicht ein, und bei dem Wärter L. machte sich eine solche durch eine kurze, einige Stunden anhaltende Temperatursteigerung bis $39^{\circ} \mathrm{C}$ bemerk. bar. Diese Allgemeinreaktion fiel nach einer ruhig verbrachten Nacht in die frühen Morgenstunden. Beide geimpfte Personen waren am Tage nach der Injektion beschwerdefrei und vollkommen arbeitsfähig.

Die Prüfung des Blutserums der beiden Geimpften vor der Injektion und zehn bis vierzehn Tage nach der Injektion ergab eine Steigerung des Titers von 0,05 auf 0,001. Die Wiederholung der Prüfung nach zwei bis drei Monaten hatte dasselbe Ergebnis. Während also $0,0 \tilde{0} \mathrm{ccm}$ des Blutserums der nicht geimpften Personen Meerschweinchen von etwa $200 \mathrm{~g}$ vor sechs bis acht tödlichen Dosen virulenter Typhusbazillen geschützt hatte, schützten in der angegebenen Zeit nach der Impfung schon 0,001 vor der gleich hohen Dosis. Das ist dieselbe Steigerung der Schutzwirkung, wie sie auch nach der Injektion mit frisch bereitetem Toxin regelmáßig beobachtet werden konnte.

Das Ergebnis dieser Prüfung berechtigt zu dem Schlub, daß das unter Beobachtung der selbstverständlichen Vorsicht bereitete Toxin auch nach jahrelanger Aufbewahrung an seiner Wirksamkeit nichts einbüßt und dab es unbeschadet seiner Wirksamkeit auch im Seeklima, in tropischen und subtropischen Gegenden lange Zeit haltbar ist.

Der nach der Briegerschen Methode hergestellte Typhusimpfstoff ist ein sicheres, ungefährliches, unter allen Verhältnissen anwendbares und unveränderliches Typhusschutzmittel. 